\title{
ESTUDIO PILOTO SOBRE EL USO DE LAS REDES SOCIALES EN JÓVENES CON DISCAPACIDAD INTELECTUAL
}

\author{
PILOT STUDY ON THE USE OF SOCIAL NETWORKS IN YOUNG PEOPLE WITH \\ INTELLECTUAL DISABILITIES
}

\author{
Mㅁ del Carmen Pegalajar Palomino; mcpegala@ujaen.es \\ Ma Jesús Colmenero Ruiz; mjruiz@ujaen.es
}

Universidad de Jaén

\section{RESUMEN}

Este artículo presenta un estudio piloto en el que se analiza el uso que hacen los jóvenes con discapacidad intelectual de las redes sociales. Para ello, se utiliza una investigación cuantitativa mediante un diseño descriptivo basado en la técnica de la encuesta $(n=75)$. Los resultados obtenidos demuestran cómo los encuestados utilizan con frecuencia este tipo de medios de comunicación social para chatear con sus amigos o conocer gente con sus mismos intereses. Del mismo modo, se constata la existencia de relaciones significativas entre la presencia de malas experiencias según el perfil en el que éstos tienen configurada la red social.

Palabras clave: Redes sociales, información, comunicación, integración social, discapacidad.

\section{ABSTRACT}

This article presents a pilot study that examines the use made by young people with intellectual disabilities in social networks. To do this, we use a quantitative research using a descriptive design technique based on the survey $(n=75)$. The results show how often respondents use this type of social media to chat with friends or meet people with the same interests. Similarly, we note the existence of significant relationships between the presence of bad experiences to the profile in which they have configured the network.

Keywords: Social networks, information, communication, social integration, disability. 


\section{INTRODUCCIÓN}

En nuestra sociedad, la aparición de la tecnología digital, Internet y la web 2.0 han ayudado a introducir una nueva forma de comunicación apoyada en el uso de redes sociales online, definidas éstas como "servicios basados en la web que permiten a sus usuarios relacionarse, compartir información, coordinar acciones y, en general, mantenerse en contacto" (Orihuela, 2008 , p.2). Las redes sociales virtuales ofrecen un espacio dónde las personas pueden crear y mantener nuevas relaciones, chatear y compartir información (Kolbitsch y Maurer, 2006); en ellas, se concentran grupos de personas que comparten las mismas necesidades y problemáticas y que se organizan para potenciar sus recursos o transmitir información (Espuny, González, Lleixà y Gisbert, 2011). Los usuarios tienen la obligación de presentar un perfil con información personal básica puesta a disposición de otros usuarios y utilizada para identificar amigos en la red y añadirlos a una lista de contactos. Autores como Petruniak, Krokosky y Terry (2011, p.1) consideran cómo los medios de comunicación social "nos pueden conectar con las comunidades de todo el mundo, acelerar nuestra comprensión de temas complejos, nos ayudan a no perder de vista lo que amamos a la vez que nos recuerdan que no estamos solos".

Las redes sociales, a pesar de tratarse de un sistema de comunicación de reciente aparición, están siendo utilizadas por millones de usuarios, muchos de los cuáles han integrado dichos sitios web en su actividad diaria (Gordo, 2006; Garmendia y Garitaonandia, 2007; Boyd y Ellison, 2008; OFCOM, 2008; Rubio, 2009; Gómez, Roses y Farias, 2012). Además, su uso entre la población más joven es en la actualidad una realidad incuestionable, llegando a convertirse en referente de comunicación por Internet (Muñoz-Rivas, Navarro y Ortega, 2003; Johansson y Götestam, 2004; Bernete, 2010). Según un estudio realizado por la Fundación Pfizer (2009), el 98\% de los jóvenes españoles de entre once y veinte años es usuario de Internet. De este porcentaje, siete de cada diez afirman acceder a la red por un tiempo diario de, al menos, una hora y media.Estos datos están muy en la línea del "Estudio sobre redes sociales en Internet" (IAB, 2009) dónde se destaca cómo un $84 \%$ de los usuarios de las redes sociales hace un uso diario de las mismas; los usuarios de redes sociales comprendidos dentro del grupo de edad de 18 a 34 años son quiénes las emplean de forma diaria.

Sin embargo, el potencial para un uso inapropiado y excesivo de ciertas aplicaciones ha dado lugar a una cierta preocupación por el impacto psicológico y conductual que puede tener Internet en las personas (Blaszczynski, 2006; Echeburúa y De Corral, 2010). En este sentido, autores como Morahan-Martin y Schumacher (2000) han establecido un promedio de 8.5 horas semanales en un grupo de usuarios considerados patológicos; por su parte, Estalló (2001) sostiene que entre 7 y 14 horas semanales pueden considerarse como un uso adaptativo de Internet. No obstante, y siguiendo diversos estudios (Brener, 1997; Shapira, Goldsmith, Keck, Khosla y McElroy, 2000), parece acertado considerar que a partir de las 20 28 horas semanales estamos ante un uso excesivo de la red que generalmente conlleva una 
interferencia de la actividad social y/o escolar o bien la presencia de malestar psicológico en el individuo.

Más concretamente, y siguiendo el estudio desarrollado por la Asociación para la Investigación de los Medios de Comunicación (AIMC, 2010), un 61.7\% de los usuarios españoles participa en Facebook, seguido de Tuenti (20,8\%), Windows Live Spaces (13\%), MySpace (10.1\%), Twitter (8.7\%), Hi5 (7.7\%) y Linkedin (7.1\%). Los encuestados, además, afirman utilizar dichas redes sociales para relaciones de amistad (79.3\%), hobbies (27\%), relaciones profesionales (23.6\%) y búsqueda de pareja (3.2\%). Estos datos vienen a corroborar los aportados por Sánchez y Aguaded (2002) en las conclusiones de su investigación "Internet y los jóvenes", dónde se destaca cómo la red no sólo pretende desarrollar un sistema de comunicación para transmitir determinados conocimientos, sino que también contempla una comunicación para relacionarse. En adolescentes y jóvenes el uso más frecuente es de carácter placentero y no instrumental, predominando las aplicaciones del Messenger, las redes sociales y las webs de entretenimiento (Conde, Torres y Ruiz, 2002; Subrahmanyam, Greenfield y Brendesha, 2004; Blais, Craig, Pepler y Connolly, 2008). Además, diferentes estudios revelan la existencia de diferencias significativas en el uso de las tecnologías según el género; así, las mujeres usan Internet para comunicarse con mayor probabilidad que los varones y ellos visitan más sitios webs y juegan más online que ellas (Gross, 2004; Aranda, Sánchez-Navarro y Tabernero, 2009).

En el caso de personas con discapacidad, el uso de las tecnologías de la información y la comunicación y, más concretamente, las redes sociales juegan un papel bastante importante, al favorecer el desarrollo de la persona y mejorar su relación con su medio. Así pues, su utilización nos ofrece una serie de posibilidades tendentes a facilitar la comunicación con otras personas y con su entorno, su incorporación en la sociedad del conocimiento así como la adquisición de aprendizajes de cara a una posterior integración en el mundo laboral. Sin embargo, el aislamiento social ha venido siendo una constante en la vida de las personas con discapacidad, por lo que, el hecho de que éstas no tengan fácil acceso a las relaciones tradicionales puede desembocar en un mayor uso y dependencia de las redes sociales virtuales (Valkenburg, Peter y Schouten, 2006). No obstante, conviene observar cómo las tecnologías no son de fácil acceso e inmediato para personas con discapacidad, ni todas ellas de hecho las utilizan (Fierro, 2009). Chacón (2007, p.261) establece como la incorporación de las TICs a personas con discapacidad debe verse "como una herramienta que permita el desarrollo personal, la realización de actividades y el disfrute de situaciones desde su propia individualidad, así como para su participación plena y activa en las actividades de su entorno".

La Fundación del Seminario Iberoamericano sobre Discapacidad y Accesibilidad en la Red (SIDAR) considera que en España el acceso a Internet por parte de los discapacitados es bastante malo, pues los portales carecen de las herramientas necesarias para ser usadas por personas ciegas, sordas o con deficiencias cognitivas. Por su parte, los datos aportados en el 
Estudio General de Medios (2002) destacan la escasa penetración de Internet en la sociedad española al contar con 7.9 millones de usuarios, es decir, el $22.7 \%$ de la población mayor de 14 años; por su parte, las comunidades de Euskadi, Cataluña y Madrid lideran el ranking de acceso a Internet, situación bastante lejana a países como Italia o Alemania con índices que rondan el 38\%. Además, la Encuesta sobre uso de nuevas tecnologías y servicios de telecomunicación (2002) desvela cómo el grado de penetración de las nuevas tecnologías y, más concretamente, de la telefonía móvil, el ordenador e Internet entre las personas con discapacidad parece superar, en general, la media del conjunto de la población española; por su parte, los colectivos con mayor presencia de ordenador presentan discapacidad de tipo físico, visual y auditiva (citado en Vega, 2003). Estos datos entran en consonancia con lo aportado por la Confederación Española de Organización a favor de las Personas con Discapacidad Intelectual (FEAPS) (Instituto de Biomecánica de Valencia, 2003) quién pone de manifiesto la creación de una brecha digital y tecnológica muy importante la cual supone una evidente situación de discriminación para este sector de la población en el acceso a los bienes de la sociedad de la información.

\section{DISEÑO METODOLÓGICO}

Con este estudio piloto se pretende dar a conocer el uso que hacen los jóvenes con discapacidad intelectual de las redes sociales. Sin duda, se trata de una población muy sensible a procesos de exclusión y/o abusos de estos medios, por lo que se propone llevar a cabo un análisis para detectar cómo utiliza este colectivo dichas redes de comunicación. Así pues, tal y como afirman Prefasi, Magal, Garde y Giménez (2010, p.109): "es el momento en las TIC contribuyan a mejorar la calidad de vida de estos colectivos, su estatus de ciudadanía y la relación de las administraciones públicas con ellos y de ellos con el resto de la sociedad".

Para ello, se ha optado por un diseño metodológico basado en técnicas cuantitativas, siendo el método de investigación la encuesta descriptiva. Al tratarse de personas con discapacidad, se ha considerado más conveniente contactar con una asociación centrada en la atención a personas con discapacidad intelectual para así acceder con mayor facilidad a la muestra. La población objeto de estudio queda constituida por todos los jóvenes que durante el año 2012 participan en el servicio de Orientación e Intermediación Laboral de la Asociación Provincial Pro-Minusválidos Psíquicos de Jaén (APROMPSI), perteneciente al ámbito FEAPS, fijándose la población de jóvenes con discapacidad intelectual de este servicio en 95 sujetos. Estas personas poseen un certificado de minusvalía con diagnóstico de discapacidad intelectual y un mínimo de un 33\% de discapacidad (retraso mental leve y moderado); al estar inscritos en la oficina de empleo, todos ellos se encuentran, por tanto, en edad laboral.

Se ha usado un muestreo probabilístico aleatorio simple (Tójar y Matas, 2009) de forma que se han ido adscribiendo a la muestra todos aquellos sujetos que han contestado a los cuestionarios $(n=75)$, quedando totalmente garantizada su representatividad con un nivel de 
confianza del $95 \%$ y un error de estimación máximo de un 5\%. En dicha muestra el $52 \%$ de los encuestados son varones, frente al $48 \%$ que lo componen mujeres; de éstos un $72 \%$ tiene una edad comprendida entre los 18 y los 27 años. En el estudio participan jóvenes de la ciudad de Jaén (61.3\%) así como de la provincia (38.7\%). La inmensa mayoría ha cursado estudios básicos (84\%), aunque no haya podido acceder aún al mercado laboral (70.7\%).

Para la recogida de datos, se ha diseñado una escala específica basada en otros cuestionarios revisados en la literatura científica existente (Vílchez, López Herrero, López Moreno y Galán, 2010; Angulo, 2011). Por ello, tras seguir las fases habituales en la elaboración de este tipo de instrumentos (Morales, Urosa y Blanco, 2003) se ha llevado a cabo el proceso de validación. Para determinar la validez de contenido del cuestionario se ha optado por el desarrollo de dos métodos fundamentales: juicio de expertos y la aplicación de una prueba piloto del instrumento. En la validación de jueces, se solicitó a varios profesores especialistas en el campo de la Didáctica y Educación Especial así como de las Nuevas tecnologías aplicadas a la Educación de diferentes universidades andaluzas que analizaran el cuestionario y nos entregaran las oportunas correcciones considerando la pertinencia de las preguntas para el logro del objetivo, claridad del lenguaje empleado en su redacción, suficiencia del número de ítems y valoración global de la propuesta del cuestionario. En todo momento, se tuvo en cuenta las limitaciones de tipo intelectual, atención y lingüísticas que pueden presentar este tipo de personas, creando un instrumento lo más sencillo posible para su cumplimentación fruto de nuestra experiencia profesional en este campo.

Tras este proceso, el cuestionario quedó finalmente elaborado, estando compuesto por 19 ítems con respuestas dicotómicas (si/no) a través de los cuáles se pregunta a los jóvenes con discapacidad intelectual acerca de las redes sociales que poseen, la frecuencia y el uso que hacen de las mismas, lugar desde dónde acceden a estos medios, obstáculos o barreras que encuentran en su utilización así como la existencia o no de malas experiencias vividas a través de estas redes de comunicación. Por su parte, el estudio de fiabilidad confirma una aceptable consistencia interna en el instrumento, al alcanzar el coeficiente alfa de Cronbach el valor de 0,725 , lo que indica que la escala diseñada es muy confiable. Dichos datos se corroboran con los valores recogidos para cada uno de los ítems del instrumento, obteniéndose en todos los casos valores por encima de 0,683.

Comprobada la validez del cuestionario, se ha procedido a la recogida de datos mediante la aplicación del cuestionario a la muestra seleccionada. En dicha fase del proceso investigador, la ética ha resultado ser un factor de vital importancia. Para ello, se ha elaborado una carta informativa a las familias de los jóvenes con discapacidad que han participado en el estudio así como una autorización a cumplimentar por los tutores legales de aquellos jóvenes que estuviesen incapacitados. En todo momento se ha tenido en cuenta principios tales como: participación voluntaria, libertad de daño, molestias físicas o psicológicas y anonimato o carácter confidencial de la información. Tras una primera entrevista con el gerente de la Asociación, se contactó con los diferentes técnicos y profesionales del Servicio de 
Orientación e Intermediación Laboral de APROMPSI, quiénes nos proporcionaron el modo de acceder a la muestra. Éstos, en sus encuentros semanales con los jóvenes, fueron los encargados de facilitarles la carta informativa para sus padres y/o tutores legales así como el cuestionario, prestándole el tiempo necesario para que éstos pudiesen cumplimentarlo adecuadamente.

\section{RESULTADOS}

Los resultados obtenidos en este estudio (tabla nำ1) demuestran cómo el 89,3\% de los jóvenes encuestados tiene cuenta en alguna de las redes sociales existentes en Internet, siendo Tuenti la red social más solicitada (79.1\%), seguida de Facebook (65.7\%), Twitter (14.9\%) y Messenger (13.4\%). De ellos, un $50.7 \%$ iniciaron su actividad en la red social no hace mucho tiempo (entre 1 y 3 años), siendo tan sólo un 31,3\% los que le comunicaron a sus padres y/o tutores legales que iban a acceder a este tipo de medios de comunicación social.

Por su parte, el $80,6 \%$ de los encuestados se puede conectar a la red sin ningún tipo de restricción, es decir, cuando ellos creen conveniente. En esta misma línea, un 52,2\% de los sujetos acceden a la red sin estar acompañados por nadie; preferiblemente de algún amigo $(48,5 \%)$, de algún familiar próximo a él $(39,4 \%)$ y de los padres $(30,3 \%)$. El sitio desde donde acceden a la red es, de manera mayoritaria, desde su propia casa $(76,1 \%)$, desde la casa de algún amigo o en el móvil (16,4\% respectivamente). Además, el 65,7\% de este colectivo afirma encontrarse con dificultades, solicitando ayuda para resolver ciertas aplicaciones o utilidades de las mismas: 56,8\% dice tener dificultad para escribir un comentario en la red en la que tiene cuenta, un $52,3 \%$ no sabe con exactitud subir fotos y darlas a conocer a través de la red y, ya en menor medida, los encuestados afirman tener problemas a la hora de crearse un perfil $(22.7 \%)$ y, por último, para publicar un evento $(11,4 \%)$.

Un $67,2 \%$ utiliza este tipo de medios para chatear con sus amigos o compañeros de trabajo; un $41,8 \%$ para conocer a gente con sus mismos gustos e intereses; un $40.3 \%$ para publicar fotos o vídeos de su entorno y finalmente, un 32.8\% lo utiliza para jugar o usar diferentes aplicaciones. En un porcentaje relativamente menor, destacamos cómo estas personas con discapacidad hacen un uso de las redes sociales para crear eventos sobre algún tema de interés (17.9\%), para saber de los demás o con un interés profesional (14.9\%).

Por lo que respecta a si todas las personas que tiene agregadas en su red social es su agrado, el $73,1 \%$ de sujetos afirma que en la red sólo tiene agregados a personas que, por diversos motivos, le satisfacen. Además, para el $50,7 \%$ de sujetos encuestados, la red social le permite relacionarse con la familia y con los amigos de una forma más satisfactoria que teniéndolos en persona, frente a un $49,3 \%$ que afirma no estar de acuerdo con tal afirmación. 


\begin{tabular}{|c|c|c|c|}
\hline & Variable & $\mathbf{n}$ & Porcentaje \\
\hline ¿Tienes cuenta en alguna de & s redes sociales de Internet? & 67 & $89.3 \%$ \\
\hline & Tuenti & 53 & $79.1 \%$ \\
\hline & Facebook & 44 & $65.7 \%$ \\
\hline & Twitter & 10 & $14.9 \%$ \\
\hline & Messenger & 9 & $13.4 \%$ \\
\hline & $\mathrm{Hi5}$ & 6 & $9 \%$ \\
\hline & MySpace & 5 & $7.5 \%$ \\
\hline & WhatsApp & 4 & $6 \%$ \\
\hline & Flickr & 3 & $4.5 \%$ \\
\hline ¿Desde hace cuánto tiempo & Entre 1 y 3 años & 34 & $50.7 \%$ \\
\hline participas en estas redes & Entre 4 y 6 años & 22 & $32.8 \%$ \\
\hline sociales? & Más de 6 años & 11 & $16.4 \%$ \\
\hline Al darte de alta, ¿̇pediste per & iso a tu padre/madre o tutor legal? & 21 & $31.3 \%$ \\
\hline ¿Puedes conectarte a la red c & Iando quieras? & 54 & $80.6 \%$ \\
\hline Cuando accedes a la red, ¿̇es & s acompañado de alguien? & 32 & $47.8 \%$ \\
\hline & Amigo & 16 & $48.5 \%$ \\
\hline & Familiar & 13 & $39.4 \%$ \\
\hline & Padre/madre & 10 & $30.3 \%$ \\
\hline ¿Desde dónde accedes a & En tu casa & 51 & $76.1 \%$ \\
\hline esta red social? & En casa de tus amigos & 11 & $16.4 \%$ \\
\hline & En el móvil & 11 & $16.4 \%$ \\
\hline & En el trabajo & 8 & $11.9 \%$ \\
\hline & En un ciber & 6 & $9 \%$ \\
\hline ¿Necesitas la ayuda de alguie & para utilizar la red social? & 44 & $65.7 \%$ \\
\hline & Escribir un comentario & 25 & $56.8 \%$ \\
\hline & Subir fotos & 23 & $52.3 \%$ \\
\hline & Crear un perfil & 10 & $22.7 \%$ \\
\hline & Crear un evento & 5 & $11.4 \%$ \\
\hline $\begin{array}{l}\text { ¿Para qué utilizas las redes } \\
\text { sociales? }\end{array}$ & $\begin{array}{l}\text { Chatear con tus amigos o compañeros } \\
\text { de trabajo }\end{array}$ & 45 & $67.2 \%$ \\
\hline & Conocer gente con tus mismos gustos & 28 & $41.8 \%$ \\
\hline & $\begin{array}{l}\text { Publicar fotos/vídeos tuyos o de tu } \\
\text { familia }\end{array}$ & 27 & $40.3 \%$ \\
\hline & Uso de aplicaciones y juegos & 22 & $32.8 \%$ \\
\hline & $\begin{array}{l}\text { Crear eventos sobre algún tema de } \\
\text { interés }\end{array}$ & 12 & $17.9 \%$ \\
\hline & Saber lo que están haciendo los demás & 10 & $14.9 \%$ \\
\hline & Interés profesional & 10 & $14.9 \%$ \\
\hline ¿Todas las personas agregad & a tu red social son de tu agrado? & 49 & $73.1 \%$ \\
\hline $\begin{array}{l}\text { ¿Crees que las redes sociales } \\
\text { y amigos mejor que cara a ca }\end{array}$ & $\begin{array}{l}\text { ermiten que te relaciones con tu familia } \\
\text { a? }\end{array}$ & 34 & $50.7 \%$ \\
\hline
\end{tabular}

Tabla 1. Tabla resumen de los principales resultados del análisis descriptivo

Por su parte, los análisis de contingencia realizados a través del coeficiente Chi-cuadrado de Pearson revelan la existencia de relaciones significativas entre el perfil que presenta el usuario en estas redes y la presencia o no de experiencias negativas para el mismo: $\chi^{2}(2$, $N=67)=0.023, p<0.05$. En este caso, tal y como muestra la tabla $n=2$, un $52.5 \%$ de los usuarios encuestados afirma haber vivido alguna mala experiencia con este tipo de redes sociales, frente al $47.8 \%$ que manifiesta no haber tenido tales experiencias. En cuanto al perfil, la mayor parte de los usuarios (65.7\%) posee una o varias redes sociales abiertas sólo a sus amigos, un $22.4 \%$ abierto para todos y, finalmente, un $11.9 \%$ extensible sólo hasta amigos 
de sus amigos. Entre aquellos que han vivido malas experiencias, se observa cómo el mayor porcentaje de encuestados presenta un perfil visible sólo para sus amistades $(51.4 \%)$, viéndose representados en situaciones en las que han recibido insultos, amenazas, alguien ha subido fotos suyas sin permiso o han inventado historias sobre el adolescente. En cambio, cuando se trata de perfiles sociales abiertos a todos los usuarios o para amigos de sus amigos dicho porcentaje se reduce considerablemente, situándose en un $20 \%$ y $28.6 \%$ respectivamente.

\begin{tabular}{lcc}
\hline \multirow{2}{*}{ Perfil } & Mala experiencia con el uso de las redes sociales \\
& $\mathrm{Si}$ & No \\
\hline Abierto a todos & $10(28.6 \%)$ & $5(15.6 \%)$ \\
Sólo visible a tus amigos & $18(51.4 \%)$ & $26(81.3 \%)$ \\
Visible hasta amigos de tus amigos & $7(20 \%)$ & $1 \quad(3.1 \%)$ \\
\hline
\end{tabular}

Tabla 2. Análisis de contingencia entre el perfil y malas experiencias vividas con las redes sociales.

\section{CONCLUSIONES}

El uso de redes sociales en jóvenes con discapacidad intelectual resulta ser un hecho bastante positivo, pues se trata de medios de comunicación social que facilita las relaciones sociales de estas personas con el contexto en el que se desenvuelven. En este sentido, Marí (2006) analiza los nuevos modos de relación e interacción que se producen en la sociedad de la información mediante el uso de las Tecnologías de la Información y la Comunicación, haciendo referencia a los jóvenes o la denominada "Generación.net" como aquella parte de la población que mejor se está apropiando de los potenciales de relación y de comunicación de tecnologías como Internet. Además, el hecho de que sean Tuenti y Facebook las redes sociales con mayor aceptación puede deberse a su facilidad de uso así como que se traten de webs abiertas cuyo perfil tecnológico es sencillo; a su vez, disponen de mayores posibilidades en cuanto a aplicaciones (foros, chat, textos, vídeos...) y creación de contenidos de distinta índole (Tapia, Gómez, Herranz, Matellanes, 2010; Hernando y Romero, 2012). Sin embargo, este tipo de redes sociales no poseen herramientas para facilitar la adscripción y el seguimiento personal de cada uno de los miembros del grupo, por lo que se hace imposible conocer el uso que estas personas llevan a cabo en este tipo de redes de comunicación.

Este tipo de medios hemos de definirlos como poderosos instrumentos que favorecen las relaciones de comunicación entre las personas con discapacidad. En este sentido, Cobo y Pardo (2007) afirman que las redes sociales se han convertido en poderosos lugares de interacción entre grupos sociales, algunos cada vez más especializados, dónde es posible ir conociendo gente que comparta los mismos intereses; ello es debido a la web 2.0 y su carácter social, mediante la formación de comunidades virtuales y redes de colaboración entre pares. De igual modo, Fernández, Revuelta y Sosa (2012, p. 62) exponen cómo "estas redes sirven para favorecer la colaboración y el trabajo conjunto ya que tienen una gran capacidad para crear grupos sociales y mantener en contacto a las personas, ofreciéndoles una visión mucho más personal e informal que otras herramientas como el correo, foros, blogs, entre otros". Por ello, es evidente que las redes sociales pueden ser un vehículo para incrementar los lazos sociales y, a su vez, ayudar a potenciar las habilidades sociales que va 
desarrollando la persona (McKenna y Bargh, 1999; Zubeidat, Salinas y Sierra, 2008; Fowler y Christakis, 2009).

Además, otros estudios demuestran el lado positivo de Internet al constatar que su uso puede favorecer la autoestima y confianza del individuo, disminuir los comportamientos depresivos, la ansiedad y la soledad (Vesmarivich, Hauber y Jones, 2000; Seymour y Lupton, 2004; Bowker y Tuffin, 2007). Incluso, existen investigaciones que consideran que, en determinadas circunstancias, la comunicación mediada por ordenador puede tener carácter terapéutico; en este sentido, Caplan (2003) señala como las personas con problemas serios de sociabilidad tienden a preferir este sistema de comunicación para desarrollar sus relaciones interpersonales. A pesar de ello, algunos autores apuntan efectos secundarios o "daños colaterales" que derivan de las interacciones producidas en estas redes de comunicación. En este caso, se trata de "discapacidad social", falta de habilidades sociales o dificultad para tratar con seres humanos reales, manifestaciones todas ellas que nos hacen reflexionar acerca de aquellos que "tienen la cabeza enterrada en una marea de desktops, laptops, celulares y dispositivos que caben en la palma de la mano" (Bauman, 2007, p.30). Además, el potencial para un uso inapropiado y excesivo de internet ha dado lugar a una cierta preocupación por el impacto psicológico y conductual que este medio puede tener en las personas (Blaszczynski, 2006). Otros autores como Fogel y Nehmad (2009) plantean los niveles de riesgo asumidos o percibidos, la confianza y la preocupación por la intimidad que manifiestan los usuarios.

No obstante, se trata de un nuevo medio que utilizan muchos jóvenes para provocar daño y causar angustia entre sus iguales (Keith y Martin, 2005; García-Pérez, 2011). El aumento de este tipo de situaciones requiere que las escuelas, familias, agentes comunitarios e instituciones se impliquen y desarrollen acciones que prevengan, controlen y regulen las agresiones tecnológicas entre iguales (Walker, 2010). Asimismo, se ha de investigar ciertos usos inadecuados de las tecnologías que, bien manejadas, presentan enormes beneficios para el adolescente y su socialización e integración social (Veen, 2003; Subrahmanyam y Greenfield, 2008). Así pues, tal y como afirma Prefasi et al. (2010) se está produciendo una evolución en la concienciación por parte de los profesionales en el diseño de aplicaciones TIC así como una mayor demanda de herramientas accesibles capaces de superar la discriminación que sufre el colectivo de personas con discapacidad para su acceso.

\section{REFERENCIAS BIBLIOGRÁFICAS}

ANGULO, J.F. (coord.) (2011). Escenarios, tecnologías digitales y juventud en Andalucía. Proyecto de Excelencia. Referencia HUM-02599. Junta de Andalucía.

ARANDA, D., SÁNCHEZ-NAVARRO, J. \& TABERNERO, C. (2009). Informe sobre el uso de herramientas digitales por parte de adolescentes en España. Barcelona: UOC.

ASOCIACIÓN PARA LA INVESTIGACIÓN DE LOS MEDIOS DE COMUNICACIÓN (2010). Navegantes en la Red. Disponible en: http://www.aimc.es (consulta: 27-11-2012).

BAUMAN, Z. (2007). Vida de consumo. Madrid: Fondo de Cultura Económica. 
BERNETE, F. (2010). Usos de las TIC, relaciones sociales y cambios en la socialización de las y los jóvenes. Revista de Estudios de juventud, 8, 97-114.

BLAIS, J.J., CRAIG, W.M., PEPLER, D. \& CONNOLLY, J. (2008). Adolescents online: the impartance of internet activity choices to salient relationships. Youth Adolescence, $37,522-536$

BLASZCZYNSKI, A. (2006). Internet Use: In Search of an Addiction. International Journal of Mental Health and Addiction, 4, 7-9.

BOWKER, N. \& TUFFIN, K. (2007). Understanding positive subjectivities made possible for disabled people. New Zealand Journal of Psychology, 36, 63-71. Disponible en: http://www.psychology.org.nz/cms_show_download.php?id=107 (consulta: 10-112012).

BOYD, D. \& ELLISON, N. B. (2008). Social network sites: definition, history, and scholarship. Journal of Computer-Mediated Communication, 13, (1), 1-11. Disponible en: http://mimosa.pntic.mec.es/mvera1/textos/redessociales.pdf (consulta: 15-102012).

BRENNER, V. (1997). Psychology of computer use: XLVII. Parameters of Internet use, abuse and addiction. The first 90 days of the Internet usage survey. Psychological Reports, $80,879-882$

CAPLAN, S. (2003). Preference of online social interaction: A Theory of Problematic Internet Use and Psychosocial Well-Being. Comunication Research, 30, (6), 625-648. Disponible en: http://crx.sagepub.com/content/30/6/625.full.pdf+html (consulta: 1510-2012).

CHACÓN, A. (2007). La atención a la diversidad con medios tecnológico-didácticos. En J.A. Ortega \& A. Chacón (coords). Nuevas tecnologías para la educación en la era digital (pp. 262-278). Madrid: Pirámide.

COBO, C. \& PARDO, H. (2007). Planeta Web 2.0. Inteligencia colectiva o medios fast fod. Disponible en: www.flacso.edu.mx/planeta/blog/index.php?option=com... (consulta: 12-9-2012).

CONDE, E., TORRES, E. \& RUIZ, C. (2002). El nuevo escenario de internet: las relaciones parasociales en adolescentes y jóvenes en la red. Cultura y Educación, 14 (2), 133-146.

ECHEBURÚA, E. \& DE CORRAL, P. (2010). Adicción a las nuevas tecnologías y a las redes sociales en jóvenes: un nuevo reto. Adicciones, 22 (2), 91-96. Disponible en:http://www.adicciones.es/files/91-96\%20editorial\%20echeburua.pdf (consulta: 312-2012).

ESPUNY, C.; GONZÁLEZ, J.; LLEIXÀ, M. \& GISBERT, M. (2011). Actitudes y expectativas del uso educativo de las redes sociales en los alumnos universitarios. Revista de Universidad y Sociedad del Conocimiento, 8 (1), 171-185. Disponible en: http://rusc.uoc.edu/ojs/index.php/rusc/article/viewArticle/v8n1-espuny-gonzalezIleixa-gisbert (consulta: 10-11-12) 
ESTALLÓ, J.A. (2001). Usos y abusos de Internet. Anuario de Psicología, 32, 95-108. Disponible en: http://www.raco.cat/index.php/anuariopsicologia/article/viewFile/61671/88438 (consulta: 15-10-12).

FERNÁNDEZ, Mạ R., REVUELTA, F.I. \& SOSA, Mạ J. (2012). Redes sociales y microblogging: innovación didáctica en la formación superior. Revista Latinoamericana de Tecnología Educativa, 11 (1), 61-74.

FIERRO, A. (2009). Retos en investigación y tecnología educativas en el ámbito de las discapacidades. Siglo Cero, 40 (1), 79-92.

FOGEL, J. \& NEHMAD, E. (2009). Internet social network communities: Risk taking, trust and privacy concerns. Computer in Human Behavior, 25 (1), 153-160.

FOWLER, J.H. \& CHRISTAKIS, N.A. (2009). The dynamic spread of happiness in a large social network: longitudinal analysis over 20 years in the Framingham heart study. British Medical Journal, 337, 1-9.

FUNDACIÓN PFIZER (2009). La juventud y las redes sociales en Internet. Madrid: Fundación Pfizer. http://www.asociacionplazadelcastillo.org/Textosweb/INFORME_FINAL_Encuesta_Ju ventud_y_Redes_Sociales.pdf (consulta: 15-10-12).

GARCÍA-PÉREZ, R. (2011). Violencia y victimización en la adolescencia: la perspectiva de los adolescentes. Tesis Doctoral: Universidad de Sevilla.

GARMENDIA, M. \& GARITAONANDIA, C. (2007). Cómo usan Internet los jóvenes: hábitos, riesgos y control parental. Informe de la investigación. Disponible en: http://www.ehu.es/eukidsonline/informe\%20final-internet.pdf (consulta: 25-102012).

GÓMEZ, M., ROSES, S. \& FARIAS, P. (2012). El uso académico de las redes sociales en universitarios. Comunicar, 38, (19), 131-138. Disponible en: www.revistacomunicar.com/pdf/preprint/38/14-PRE-13426.pdf (consulta: 25-102012).

GORDO, A.J. (2006). Jóvenes y cultura Messenger. Tecnología de la información y la comunicación en la sociedad interactiva. Madrid: INJUVE. Disponible en: http://www.fad.es/sala_lectura/Messenger.pdf (consulta: 15-10-2012).

GROSS, E.F. (2004). Adolescent internet use: what we expect, what teens report. Developmental Psychology, 25, 633-649. Disponible en: http://www.sciencedirect.com/science/article/pii/S0193397304000772 (consulta: 15-10-2012).

HERNANDO, A. \& ROMERO, M.I. (2012). Redes sociales y ciudadanía en las nuevas $\begin{array}{lllll}\text { sociedades } \quad \text { participativas. } & \text { 27-30. }\end{array}$ http://www.aularia.org/ContadorArticulo.php?idart=13 
IAB (2009). Estudio sobre redes sociales en Internet. Disponible en: http://www.ontsi.red.es/ontsi/sites/default/files/redes_sociales-documento_0.pdf (consulta: 15-9-2012).

INSTITUTO DE BIOMECÁNICA DE VALENCIA (2003). Libro Blanco $1+D+l$ al servicio de las personas con discapacidad y las personas mayores. Valencia.

JOHANSSON, A. \& GÖTESTAM, K.G. (2004). Internet addiction: characteristics of a questionnaire and prevalence in Norwegian youth (12-18 years). Scandinavian Journal of Psychology, 45, 223-229.

KEITH, S. \& MARTIN, M.E. (2005). Cyberbullying: Creating a culture of respect in a cyber world. Reclaiming Children and Youth, 13, 224, 228.

KOLBITSCH, J. \& MAURER, H. (2006). The transformation of the Web: How emerging communities shape the information we consume. Journal of Universal Computer
Science,
2
(2),
187-213.
Disponible
en: http://www.jucs.org/jucs_12_2/the_transformation_of_the/jucs_12_02_0187_0214 _kolbitsch.pdf (consulta: 25-9-2012).

MARÍ, V.M. (2006). Jóvenes, tecnologías y el lenguaje de los vínculos. Comunicar, 27, 113116. Disponible en: http://redalyc.uaemex.mx/pdf/158/15802717.pdf (consulta: 2011-2012).

MCKENNA, K. \& BARGH, J. (1999). Causes and consecuences of social interaction on the Internet: a conceptual framework. Media Psychology, 1, 249-261.

MORAHAN-MARTIN, J. \& SCHUMACHER, P. (2000). Incidence and correlates of pathological Internet use among college students. Computer in Human Behavior, 16, 13-29.

MORALES, P., UROSA, B. \& BLANCO, A. (2003). Construcción de escalas de actitudes tipo Likert. Una guía práctica. Madrid: La Muralla.

MUÑOZ-RIVAS, M.J., NAVARRO, M.E. \& ORTEGA, N. (2003). Patrones de uso de Internet en población universitaria española. Adicciones, 15, 137-144. Disponible en: http://www.adicciones.es/files/04-Patrones\%20uso\%20internet.pdf (consulta: 20-92012).

OFCOM (2008). Social networking: A quantative and qualitative research report into attitudes, behaviours, and use. Disponible en: www.ofcom.org.uk/advice/media_literacy/medlitpub/medlitpubrss/socialnetworking /report.pdf (consulta: 17-10-2012).

ORIHUELA, J. L. (2008). La hora de las redes sociales. Nueva Revista de Política, Cultura y Arte, 119, 57-62.

PETRUNIAK, M., KROKOSKY, A. \& TERRY, S. (2011). Social media provides tools for discovery: how to find value in social networking. The Exceptional Parent. Disponible en: http://www.thefreelibrary.com/Social+media+provides+tools+for+discovery\%3A+ho w+to+find+value+in...-a0268310914 (consulta: 20-5-2012). 
PREFASI, S., MAGAL, T., GARDE, F. \& GIMÉNEZ, J.L. (2010). Tecnologías de la Información y la Comunicación orientadas a la educación de personas con discapacidad cognitiva Revista Latinoamericana de Tecnología educativa, 9 (2), 107-123.

RUBIO, A. (2009). Adolescentes y jóvenes en la red: factores de oportunidad. Madrid: INJUVE. Disponible en: http://www.adideandalucia.es/documentos/infeducativa/adolescentesenlared.pdf (consulta: 15-10-2012).

SÁNCHEZ, M. \& AGUADED, I. (2002): Internet y los jóvenes. Granada-Huelva: Grupo Comunicar.

SEYMOUR, W. \& LUPTON, D. (2004). Holding the line online: Exploring wired relationship for people with disabilities. Disability and Society, 19, 291-305.

SHAPIRA, N.A., GOLDSMITH, T.D., KECK, P.E., KHOSLA, U.M. \& MCELROY, S.L. (2000). Psychiatric features of individuals with problematic Internet use. Journal of Affective Disorders, 57, 267-272. Disponible en: http://www.sciencedirect.com/science/article/pii/S016503279900107X (consulta: 15-10-2012).

SUBRAHMANYAM, K. \& GREENFIELD, P. (2008). Online communication and adolescent relationships. The future of Children, 18, 119-146.

SUBRAHMANYAM, K., GREENFIELD, P.M. \& BRENDESHA, T. (2004). Constructing sexuality and identify in an online teen chat room. Applied Developmental Psychology, 25, 651$666 . \quad$ Disponible en: https://www.centerschool.org/pko/documents/Constructingsexuality.pdf (consulta: 15-10-2012).

TAPIA, A., GÓMEZ, B., HERRANZ, J.M. \& MATELLANES, M. (2010). Los estudiantes universitarios ante las redes sociales: cuestiones de uso y agrupación en estructuras elitistas o pluralistas. Vivat Academia, 113. Disponible en: http://www.ucm.es/info/vivataca/numeros/n113/DATOSS.htm (consulta: 15-102012).

TÓJAR, J.C. \& MATAS, A. (2009). Fundamentos metodológicos básicos. En A. Pantoja (coord.). Manual básico para la realización de tesinas, tesis y trabajos de investigación (pp.129-154). Madrid: EOS.

VALKENBURG, P.M., Peter, J. \& Schouten, A.P. (2006). Friend networking sites and their relationship to adolescents well-being and social self-esteem. CyberPsychology \& Behavior, 9, 584-590.

VEEN, W. (2003). A new force for change: Homo zappiens. The Learning Citizen, 7, 5-7.

VEGA, A. (2003). Internet para todos: en el Año Europeo de la Discapacidad. XXI, Revista de Educación, 5, 13-26. Disponible en. http://www.uhu.es/publicaciones/ojs/index.php/xxi/article/viewArticle/639 (consulta: 15-10-2012). 
VESMARIVICH, S., HAUBER, R.P. \& JONES, M. (2000). Using telecommunication technologies to change the world for people with disabilities related to catastrophic neurological impairment. CyberPsychology \& Behavior, 3, 925-928.

VÍLCHEZ, L.F., LÓPEZ, M., LÓPEZ, V. \& GALÁN, E. (2010). Redes sociales de internet y adolescentes. $\quad \mathrm{La}$ dimensión social. Disponible en: http://www.websatafi.com/joomla/attachments/125_REDES\%20SOCIALES\%20PUBLI CAR.pdf (consulta: 5-10-2012).

WALKER, H.M. (2010). Relational aggression in schools: implications for future research on screening intervention and prevention. School Psychological Review, 38, 594-601. Disponible en: http://www.nasponline.org/publications/spr/39-4/spr394walker.pdf (consulta: 15-10-2012).

ZUBEIDAT, I., SALINAS, J. \& SIERRA, J. (2008). Evaluación de factores asociados a la ansiedad social y a otras psicopatologías en adolescentes. Clínica y salud, 31, 189-196. Disponible en: http://www.scielo.org.mx/scielo.php?pid=S018533252008000300004\&script=sci_art text (consulta: 18-9-2012).

\section{Para citar este artículo:}

Pegalajar, M.C. \& Colmenero, M.J. Estudio piloto sobre el uso de las redes sociales en jóvenes con discapacidad intelectual. EDUTEC, Revista Electrónica de Tecnología Educativa, 